\title{
IOT BASED SYSTEM FOR DETECTION OF SEWAGE BLOCKAGES
}

\author{
Pratim Bhosale ${ }^{1}$, Shivani Mithapelli ${ }^{2}$, Shivani Bollabattin ${ }^{3}$, Rucha Patel ${ }^{4}$, \\ Asim Bhalerao $^{5}$ \& Dr. Dipti D. Patil ${ }^{6}$ \\ ${ }_{1}$ Dept. of Information Technology Cummins College of Engineering \\ pratim.bhosale@cumminscollege.in \\ ${ }_{2}$ Dept. of Information Technology Cummins College of Engineering \\ shivani.mithapelli@cumminscollege.in \\ ${ }_{3}$ Dept. of Information Technology Cummins College of Engineering \\ shivani.bollabattin@cumminscollege.in \\ ${ }_{4}$ Dept. of Information Technology Cummins College of Engineering \\ rucha.patel@cumminscollege.in \\ ${ }_{5}$ Founder of Fluid Robotics Pvt. Ltd. \\ asim@fluidrobotics.com
}

${ }_{6}$ Associate Professor Cummins College of Engineering dipti.patil@ cumminscollege.in

\begin{abstract}
In smart cities, the Internet of Things(IoT) is a key aspect providing seamless and unique access to public resources, with the aim of achieving better utilization and optimization of power, water and transportation facilities. Today urban India is faced with a water crisis, about $80 \%$ of sewage enters lakes, rivers and groundwater untreated, due to the lack of effective wastewater infrastructure management. This research focuses mainly on monitoring of sewage infrastructure, to predict blockages, preventing them from turning into Sanitary Sewer Overflows (SSO). This paper proposes an IoT based full-fledged system to tackle this issue. The system monitors the working of access manholes in a network, collecting multiple data parameters, generated in and around these manholes, and sends alerts when the development of a blockage is detected. This geospatial data along with the alerts are observed via a centralized visual dashboard. Through this system, the objectives that the paper proposes to achieve, are generation of dynamic geo-spatial map of
\end{abstract}

manhole networks, enabling the stakeholders to perform proactive maintenance of this

infrastructure. Another goal of this work is to generate a data set for developing machine learning models for advanced maintenance methods.

Keywords: sewer blockage detection; manhole; smart cities; IoT; wastewater maintenance; sanitary sewer overflow; LoRaWAN; ultrasonic sensor; gas sensor; inertial measurement unit;

\section{Introduction}

To empower India digitally the Government of India has launched many programs. Under the Digital India program, the Ministry of Urban Development has shown preference towards the use of IoT while developing Smart City plans, to enable their overall strategy and overcome operational and community challenges [1]. Hence Smart Cities Mission is one of the major initiatives under the Digital India program. Its 
main goal is to provide people with a better quality of life.

During monsoon season, rainwater seeps into these ill-maintained manholes, drastically increasing sewage levels. Such incidents stall the routine of the city on a severe level and sometimes also leads to several infectious diseases. Blockages developing in sewer pipelines go undetected for years. They are detected only when sewage starts overflowing onto the streets through the manhole i.e. once the blockage has progressed to a stage where it may be beyond repair. This condition, commonly referred to as Sanitary Sewer Overflow (SSO) [2] are observed at a stage where they are extremely difficult to fix. In severe scenarios where the blockages cannot be repaired, a bypass has to be constructed which is an expensive affair, and often results in the sewage directly entering our water ways. The prevailing mechanisms include use of jetting/ suction [3] to loosen blockage, bucket machines to loosen blockage, build a bypass route or sometimes replace the entire blocked line.

IoT has been a remarkable contributor towards smart city solutions. A critical need to consider when developing a smart city is smart underground infrastructure. Monitoring of the sewerage and drainage system plays a vital role in keeping the city healthy and functioning. However, current processes involving human interventions leads to ineffective and error prone management of sewers, resulting in delayed and expensive handling of solutions. Sanitary Sewer Overflows also have a significant impact on the health of groundwater, lakes and rivers, in turn affecting the health of citizens. Therefore, the need for a smart sewage monitoring system to predict blockages and avoid overflows has never been higher.

\section{Literature Survey}

There are various news articles related to SSOs and deaths occurring due to poor maintenance techniques. It has been noted that more Indians die while performing sewer maintenance than while fighting along the borders [4]. Around 814 sewer deaths have been recorded between 1993 to
2019, whereas unofficial numbers are expected to be significantly higher [5]. Recurring flood conditions have also brought to light the poor state of sewage and drainage infrastructure.

SSOs cause serious disruption in the daily routine of citizens. Also response to complaints is often delayed and sometimes even left unattended by Municipal Corporation due to lack of as-built maps of the manhole networks [6]. The underground sewerage system is designed to collect and drive out all the liquid waste from homes and industries. Sewerage is the system which involves elements such as, access manholes, pipe sections, pumping stations, flood overflows, mixed sewage or sanitary sewer screening chambers, and treatment works [7].

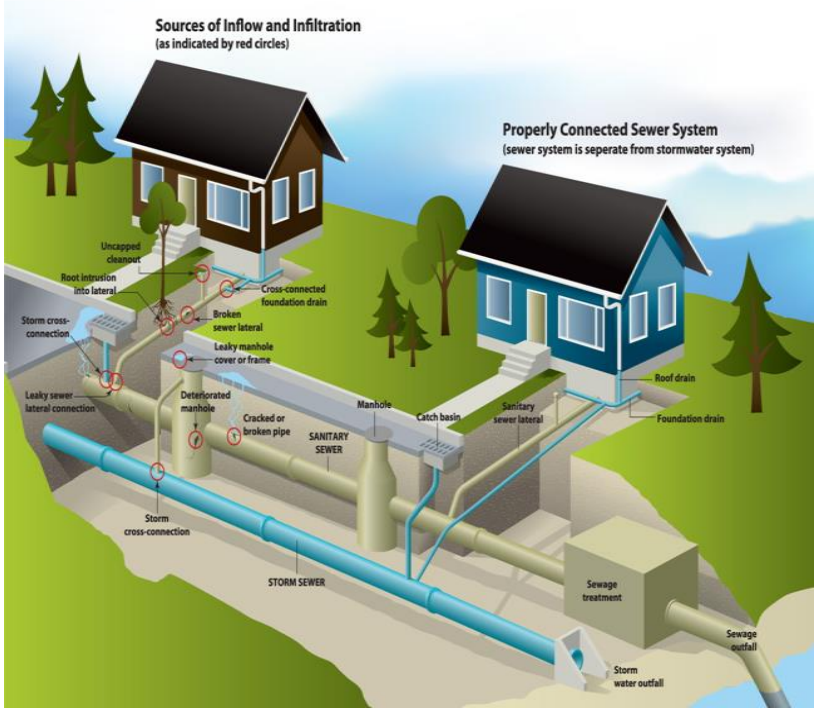

Figure 1. Sewer Network [7]

The Figure 1 depicts general layout of a sewage system. The sewage outlets from the households of a particular area are connected to the trunk main of that locality. This central pipeline has access manholes, spaced roughly every $30 \mathrm{~m}$ for maintenance access. Through these manholes a person could enter a sewer for examination, cleaning and perform various other maintenance tasks. The manhole is fitted with a robust removable lid that can 
withstand traffic loads [8]. The manholes are first constructed before the sewers are set. The manhole can have multiple incoming and outgoing pipe connections, through which the wastewater flows. By design the pipelines have a gentle slope which facilitates easy flow of liquid waste [9]. The various parameters that the paper proposes to study are: width and depth of the manhole, incoming and outgoing pipe connections with dimensions, benching on each side and the manhole base slab. The depth of manhole is calculated using Manning's formula [10]. To maintain downstream flow unanimously pumping stations are built in low lying areas. This architecture expands across cities and forms the entire network of sewage.

Many approaches have been suggested for the management and control of the sewage system, including the use of cameras and the installation of sensors in the manholes. The literature [11] offers a summary of a system that tracks the blockages occurring between two manholes additionally sensing a rise in water levels and varied gases, potentially harmful to humans. The system then triggers an alarms, providing Health Departments with critical data. Another low- cost, and lowmaintenance solution in [11] provides IoT based real-time notifications to management stations via email when any manhole exceeds its threshold values. An approach has been presented for strengthening Shoalhaven Water (SW) Departments wastewater network management procedures. SW is the local authority for water management in New South Wales, Australia. It is one of the first to supply testing of network capabilities of the Long Range Wide Area Network (LoRaWAN) in Australia [12]. It is found that within a specified geographical area the use of LoRaWAN protocol can achieve maximum transmission distances of $20 \mathrm{~km}$ and up to $35 \mathrm{~km}$ of visibility in an urban environment.

This paper addresses many important challenges regarding existing drainage systems, including negligence of manhole maintenance, lack of real-time maps of sewage networks, delayed actions in the event of serious damage, with minimal presence of IT solutions in this domain. Many municipal corporations [4] have area wise Nala Basin maps for Sewage lines only. But since these are static maps, with no regular updates to the database they are not useful for critical decision making. Further, less emphasis has been given on manhole specific maps. Even with the presence of several IT solutions, how many of these are sensor based with real- time monitoring inside manholes? Hardly any

\section{Proposed Work}

In the proposed system, the device will be installed inside a manhole chamber on the side/vertical wall. The device inside a manhole will consist of a microprocessor, a level sensor, a gas sensor array and an IMU. The ultrasonic sensor will record continuous measurements of wastewater levels [13]. Drastic changes in levels will be one of the parameters that will help trigger a red flag. Also an $\mathrm{H} 2 \mathrm{~S}$ gas sensor will continuously monitor for $\mathrm{H} 2 \mathrm{~S}$ gas and a concentration close to $100 \mathrm{ppm}$ will be caught in the logic to further trigger an alert [14]. The IMU shall monitor the device health. The threshold values for sensed parameters will be configured. If the thresholds are crossed, alerts will be triggered based on the severity. Installation of several such smart wireless devices in manholes of a particular area connected to a centralized gateway will lead to the formation of a star network. A centralized monitoring system will be developed for keeping track of the entire network. The data from all the edge devices will be directed towards a central gateway, which will further send it to a centralized monitoring system. The user can view the alerts sent from the devices through web application dashboard.

The communication in the system is through LoRa technology. LoRa is a radio modulation technology for wireless LAN networks in the category of LPWA network technologies. LoRaWAN is a network (protocol) using LoRa. Deployed in a star topology, a network based on the open LoRaWAN protocol is suitable for applications that require long- 
range or deep in-building communication among a large number of devices that have low power requirements and that collect small amounts of data. LoRa is a radio frequency carrier signal based in the physical (PHY) layer that converts the data it receives to signals. LoRaWAN is a protocol located in the Media Access Control (MAC) layer that promotes the LoRa signals to upper layers. In our system, edge devices with LoRa chips will communicate with the LoRa gateway. From the gateway, the sensed data will be directed to the cloud.

\subsection{System Architecture}

The Figure 2 depicts the proposed architecture which consists of three layers namely physical, communication and application. The first layer consists of several sensor nodes that monitor different parameters such as wastewater levels, methane and hydrogen sulfide gas concentrations, GPS coordinates to locate the manhole, accelerometer and gyroscope coordinates from the IMU to monitor the device and send alerts in case the device is unstable or dislodged. The communication layer is responsible for connecting several sensor nodes to the network and provides a medium over which data is processed and transmitted/received. The sensor nodes communicate with the gateway using LoRa protocols. The gateway acts as a controller and all sensor nodes are connected to the gateway which forms a star topology. Application layer provides an interface between end devices and network. This layer is responsible for processing, visualization and data analysis.

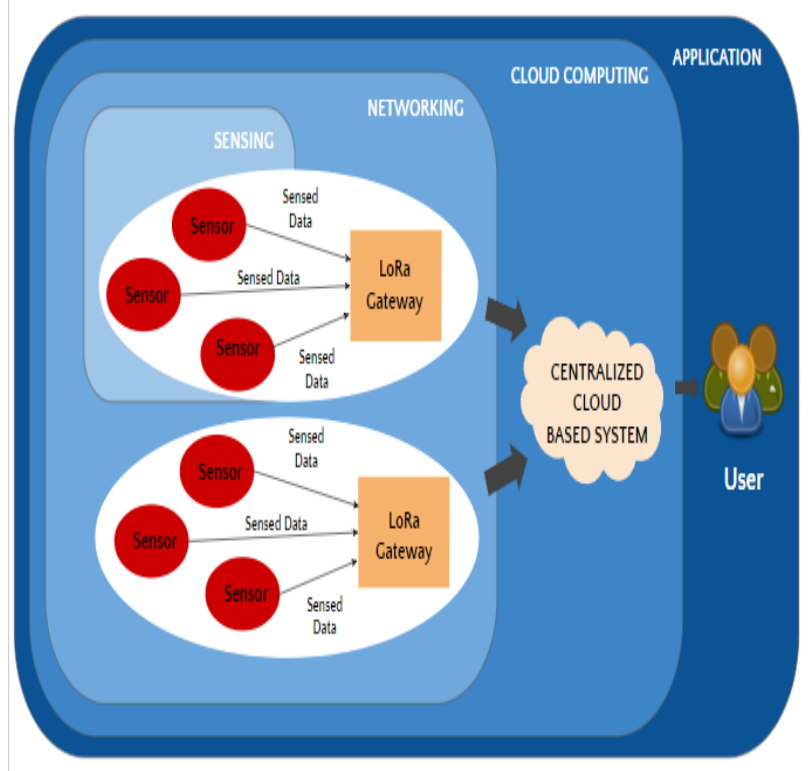

Figure 2. Layered Architecture

\subsection{System Components}

Wireless Sensor Networks(WSN) are already being used in underground drainage monitoring systems. The wireless sensor network consists of sensor nodes, communication network and cloud storage. In order to examine the data and analyze results, a web interface is developed further. The sensor nodes collect the required parameters processed by an onboard computer, which sends the data to the centralized system through the communication network.

\subsubsection{Ultrasonic Sensor:}

Ultrasonic sensors are well-known for their robust performance in dusty and harsh environments or where precise detection is the main target. They are unaffected by target color, noise or dirt conditions and are effective in detecting and monitoring objects. Ultrasonic sensors are nonintrusive in which they require no physical contact with their target. An ultrasonic sensor consists of a transmitter and receiver which is able to transmit and receive the ultrasonic sound. It discharges short, highrecurrence sound heartbeats at standard intervals [15]. It detects the space by transmitting an ultrasonic wave which travels within the air and when it gets obstructed by any material, it reflects back 
to the receiver sensor. The separation between the target and the sensor is calculated based on the time-range starting from the emitted signal to the receiving echo [16]. The measurement range of these sensors is up to several meters. Utilizing this sensor, the change in the level of water can be estimated. Ultrasonic sensors have a variety of applications such as robot navigation, measurement of air-flow velocity, anti-collision detection, vehicle detection in barrier systems, level measurement, etc. [17]

\subsubsection{Gas Sensor:}

Gas sensors measure the concentrations of harmful gases present in the environment. The sensors consist of a small heater inside, with an electro-chemical sensor which measures different kinds of gas concentrations. According to the concentration detected, the gas sensor creates a relating potential difference by changing the resistance of the material inside the sensor, which can be estimated as output voltage [16]. Based on this voltage value, the type and grouping of the gas can be evaluated. These types of sensors can recognize a variety of gases, in our case, hydrogen sulfide and methane present inside sewer vents. The concentration of hydrogen sulfide inside the manholes ranges from as low as $2 \mathrm{ppm}$ to high concentrations up to $100 \mathrm{ppm}$. Hydrogen Sulfide gas can be toxic even in low concentrations, and can be fatal. H2S gas under certain conditions can convert to sulfuric acid that attacks concrete and steel. [18]

\subsubsection{Inertial Measurement Unit:}

Inertial Measurement Unit is a unit that gives the physical direction of the device subject to three axes - gyroscope 3 axes, accelerometer 3 axes, magnetometer 3 axes which in total gives 9 degrees of freedom. These sensors are utilized for movement tracking and analysis. Accelerometers evaluate the direction and rate of change of velocity. Gyroscopes measure the angular velocity. Magnetometer finds the direction of the gadget based on the earth's magnetic field. It measures the device's location in three-dimensional space giving directional information, position, speed, and course of development, at a given time in space utilizing the IMU [19]. These sensors are normally used in robot navigation.

\subsubsection{LoRa:}

LoRa (long range) is a network layer protocol which uses a spread spectrum modulation technique derived from chirp spread spectrum (CSS) technology. It uses the unlicensed frequency bands $433 \mathrm{MHz}$ and $868 \mathrm{MHz}$ for connectivity. They are highly preferred in the long range transmission up to $15 \mathrm{Kms}$. Devices capable of adapting LoRa technology and connecting to the open LoRaWAN protocol enable smart IOT applications which can overcome some serious challenges: Energy management, depletion in natural resources, pollution control, infrastructure inefficiency. This is a network layer protocol which is designed for low bit rate but long range and low power applications. The communication between the end node and gateway is bidirectional allowing the end node to receive data from the gateway. Due to its unique modulation, LoRa is an efficient technology that can be adapted to different environment types and application classes [20]. LoRa technology itself incorporates GSM and GPS functionalities. Keeping in mind the conditions of a manhole, LoRa is best suited to penetrate through the concrete lids.

In the proposed system, microprocessor unit (MCU) records the collected data to be transmitted to the communicating nodes through a wireless, unidirectional radio frequency communication. To help achieve energy autonomy, the sensor nodes will be as low power as possible and as simple as possible. The Table 1 clearly shows how the data flow rate, range, power consumption and frequency will meet our requirements.

Table 1. Comparison of loT Protocols

\begin{tabular}{|l|l|l|l|l|}
\hline $\begin{array}{l}\text { Points of } \\
\text { Difference }\end{array}$ & Wi-Fi & ZigBee & LoRa & 4G/5G \\
\hline Data Rate & $10-$ & $20-50$ & $0 / 3-50$ & 200 \\
\hline
\end{tabular}




\begin{tabular}{|l|l|l|l|l|}
\hline & $\begin{array}{l}100+ \\
\text { Mbps }\end{array}$ & Kbps & Kbps & M \\
\hline Range & $100 \mathrm{~m}$ & $\begin{array}{l}10- \\
100 \mathrm{~m}\end{array}$ & $100 \mathrm{~km}$ & $\begin{array}{l}\text { S } \\
\text { m }\end{array}$ \\
\hline $\begin{array}{l}\text { Power } \\
\text { Usage }\end{array}$ & Medium & Low & Low & M d \\
\hline Frequency & $\begin{array}{l}2.4-5 \\
\text { GHz }\end{array}$ & $\begin{array}{l}2.4 \\
\text { GHz }\end{array}$ & $\begin{array}{l}\text { Sub } \\
\text { GHz }\end{array}$ & $\begin{array}{l}\text { C } \\
\text { B }\end{array}$ \\
\hline Cost & Low & Medium & Medium & Hig \\
\hline
\end{tabular}

\subsubsection{Cloud:}

ThingSpeak is an open-source IoT application and an API for storing and retrieving data from things over the Internet or through a local area network using the HTTP and MQTT protocols. Using MQTT, the sensor data is forwarded to the thingspeak server. MQTT publishes the sensor data on the thingspeak channel. It visualizes the data in real-time. Using MATLAB it allows to analyze the data. For Prototyping purpose, the system uses Thingspeak cloud for data visualization. In actual implementation the author proposes to use Google Cloud Platform (GCP). GCP's IoT Core is a completely managed service that lets you easily connect, manage, and extract data from millions of globally distributed devices. In conjunction with other services on GCP, IoT Core provides a complete solution for the collection, processing, analysis and visualization of IoT data in real time to support improved operational efficiency. The real time sensor data will be streamed on cloud Pub/Sub via gateway. Google Cloud Pub/Sub is a messaging function asynchronous in nature which enables the exchange of messages between autonomous applications. These messages are then imported using Google BigQuery for storage and data visualizations.

\subsection{Implementation}

The system can be well mapped with a level 5 IoT architecture and can be explained by studying the various IoT protocols used on different layers. Multiple sensing device (end nodes) along with the LoRa gateway (coordinator node) forms the base of level 5
Mbps Iof architecture. Communication between the end device and gateway is achieved via SeveraLoRa. The data collected from end nodes miles will be sent to cloud via coordinator node Mediuthprough 4G. Data is stored and analyzed in the cloud and application is cloud based. CellulaMaking the application cloud based will Bands help in management of BigData. Wide range of protocols are available on each layer. On application layer subscription to the data from the devices is done using the MQTT protocol. MQTT architecture contains three components. A publisher, a broker, and a subscriber [21]. The gateway will act as an MQTT broker. The centralized system registers on it as a subscriber to be informed when the publisher devices publish his topics by the broker. The publisher transfers the information to the subscribers via the broker. Transmission control protocol(TCP) is mostly used on transport layer. It makes sure that it keeps a log of packets transmitted and takes care of retransmission of the lost packets while maintaining steady flow control. But for an embedded system like this, TCP can be an overfit. Therefore, UDP can be considered as the best option in this case for data transfer as it does not waste overhead space. The network layer protocol 6LoWPAN and Data link layer protocol 802.15.4-LR-WPAN are best suited for LoRa technology.

\section{Results}

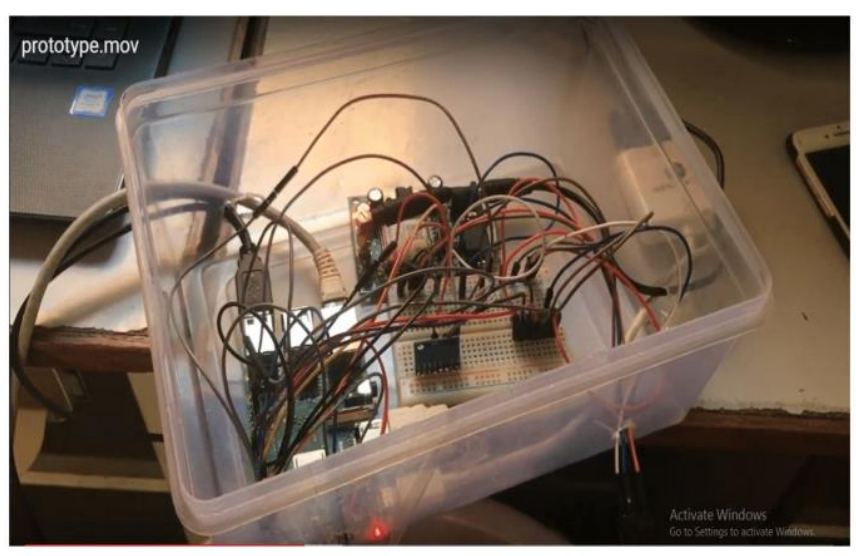

Figure 3. Prototype

The above image shows implemented prototype using Raspberry Pi. This prototype demo was conducted by 
simulating a manhole chamber. The water level readings were measured in centimeter. The ultrasonic sensor - HC-SR04, Methane gas sensor - MQ4, Inertial measurement unit - MPU6050 and a GSM SIM800A module are interfaced with a Raspberry Pi. A laptop is configured as a gateway between the Raspberry Pi and cloud.

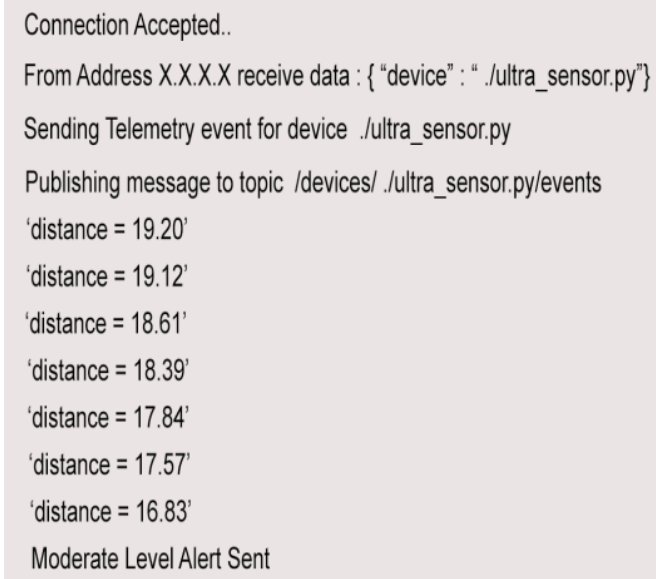

\section{Figure 4. Gateway Snapshot}

Figure 4 shows the telemetry data snapshot of the gateway. A particular instance of data transfer consists of Internet protocol (IP) address of the source node, device specification and the actual data readings.

For prototyping purpose, the LoRa features of real-time messaging and positioning have been fulfilled using a low cost GSM module. Whenever the parameter thresholds are crossed an SMS alert is sent to the official's mobile through the GSM Module5. Critical and moderate level alerts will be sent to the administrator as per change in the parameters,

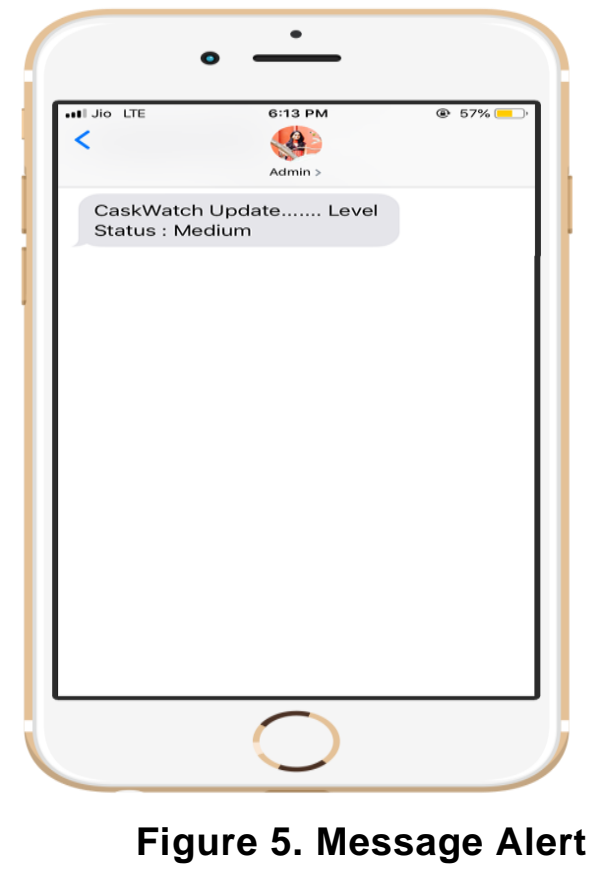

The Figure 6 shows the real time web based dashboard for the system. It consists of graphs for ultrasonic sensor with data points as different distance readings, gas level concentrations inside manhole for gas sensor and stability coordinates in case of IMU. These will help us monitor all the parameters for a particular device. One can have an overview of the entire network by looking at the map of actual site. The map in the Figure 7 is developed by plotting actual data points gathered from datasets of sewer pipelines and manholes in Powai, Mumbai. Each blue point represented on the map is a manhole and the dark line between two points is a sewer pipeline connecting two manholes. The interactive map enables the administrator to get an overview off the whole area. The system will show visual alerts regarding node failure, low battery or device complications via the maps. 


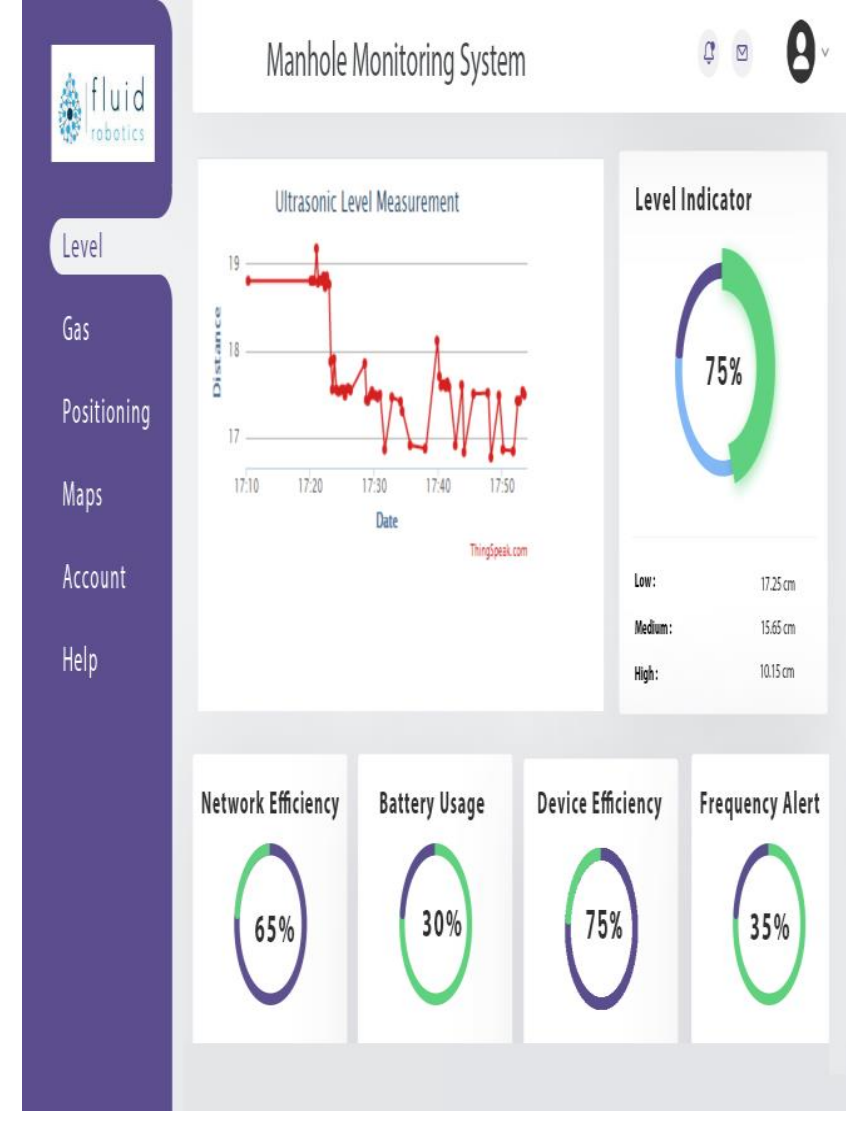

Figure 6. Water Level Monitoring

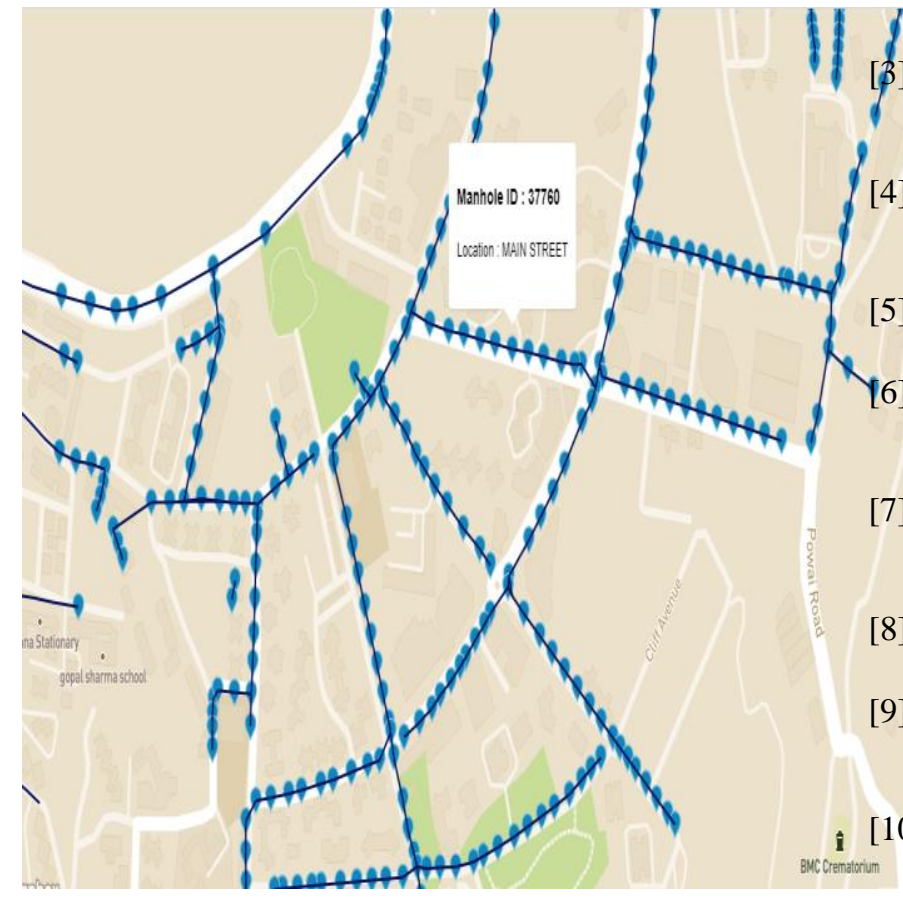

Figure 7. Manhole and Sewer Line Map

\section{Conclusion}

The existing systems have high manual interventions which can be eliminated by having Smart solutions for sewage systems will be more beneficial in terms of more effective and data driven decision making. It will also avoid severe damage and help in quick identification of the potential risks in early stages. Implementing this system on real site, can help gather data which will be helpful in calibrating the device. The findings of this study are expected to further encourage development and production of highly scalable and resilient devices. The major objective of enabling the stakeholders to perform proactive maintenance of sewage infrastructure is achieved. With the help of dynamic geospatial maps, monitoring of manhole network has become easier. Thus fulfilling the goal of providing a cleaner and safer living place to the people is achieved.

\section{References}

[1] Ministry of Electronics and Information Technology, About Digital India, 2019.

[2] T. Nasrin, A. K. Sharma, and N. Muttil, "Impact of short duration intense rainfall events on sanitary sewer network performance," Water, vol. 9, no. 3, p. 225, 2017.

3] Government of India, Ministry of Housing and Urban Affairs, Standard operating procedure (sop) for cleaning of sewers and septic tanks, 2018

[4] QuartzIndia, Ajaz Ashraf, "More Indians die cleaning sewers than fighting terrorists in Kashmir," 2017. [Online].

[5] National Commission for Safai Karamcharis, Sewer death cases, 2019.

16] Pune Municipal Corporation, "Sewage treatment plants," 2019. [Online]. Available: https://pmc.gov.in/en/drainagel.

[7] Banisheraton, Cost to hook up to city sewer ct, how much does it cost to connect to a public sewer, 2019.

[8] msd Safe Clean Waterways, What happens after you flush, 2012.

[9] Government of India Ministry of Housing and Urban Affairs, Design and Construction of Sewers, 2019

[10] L. Bogdan, G. Petriczek, and J. Studzinski, "Mathematical modeling and computer aided planning of communal sewage networks," Journal of Automation Mobile Robotics and Intelligent Systems, vol. 8, 2014.

[11] SONAWANE, GAURANG and MAHAJAN, CHETAN and NIKALE, ANUJA and DALVI, YOGITA, "Telemetry channel codingsmart realtime drainage monitoring system using internet 
of things," 2018.

[12] A. Drenoyanis, R. Raad, I. Wady, and C. Krogh, "Implementation of an iot based radar sensor network for wastewater management," Sensors, vol. 19, no. 2, p. 254, 2019

[13] . H. Tadokoro, P. Jp, N. Nakamura, T. Nishimura, K. Uemura, N. Kikuchi, and M. Hatayama, "Monitoring and control systems for the iot in the water supply and sewerage utilities".

[14] R. Stopforth and S. Davrajh, "Gas concentration and equation correlation: Of the figaro sensors, used for dangerous environments," IEEE AFRICON, pp. 1420-1425, IEEE, 2017.

[15] N. Asthana and R. Bahl, "Iot device for sewage gas monitoring and alert system," in 1st International Conference on Innovations inInformation and Communication Technology (ICIICT), pp. 1-7, IEEE, 2019.

[16] R. Girisrinivaas and V. Parthipan, "Drainage overflow monitoring system using iot (doms)," in IEEE International Conference on Power, Control, Signals and Instrumentation Engineering (ICPCSI), pp. 2133-2137, IEEE, 2017.

[17] M. Kelemen, I. Virgala, T. Kelemenova, L. Mikova, P. Frankovsk' y, 'T. Liptak, and M. L orinc, "Distance measurement via using of ultrasonic sensor," Journal of Automation and Control, vol. 3, no. 3, pp. 71-74, 2015.

[18] P. SŁ strand, B. r. Tvedt, W. Eduard, E. Bye, and K. Heldal, "Hazardous peak concentrations of hydrogen sulfide gas related to the sewage purification process," AIHAJ-American Industrial Hygiene Association, vol. 61, no. 1, pp. 107-110, 2000.

[19] H. Lategahn, M. Schreiber, J. Ziegler, and C. Stiller, "Urban localization with camera and inertial measurement unit," IEEE Intelligent Vehicles Symposium (IV), pp. 719-724, IEEE, 2013.

[20] M. Bor and U. Roedig, "Lora transmission parameter selection," in 13th International Conference on Distributed Computing in Sensor Systems (DCOSS), pp. 27-34, IEEE, 2017.

[21] M. B. Yassein, M. Q. Shatnawi, S. Aljwarneh, and R. Al-Hatmi, "Internet of things: Survey and open issues of mqtt protocol," in International Conference on Engineering \& MIS (ICEMIS), pp. 1-6,IEEE, 2017.

\section{Authors}

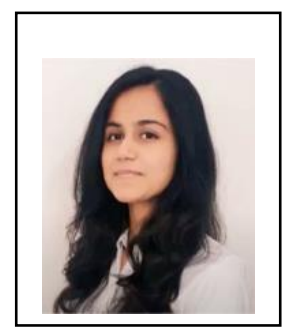

Pratim Bhosale,

Completed BTech from MKSSS's Cummins College of Engineering for Women, Pune in the year 2020 .

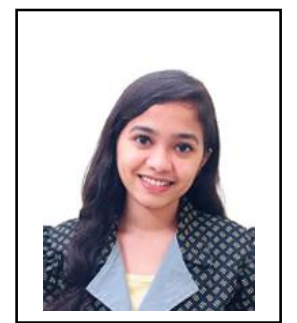

Shivani Mithapelli, Completed BTech from MKSSS's Cummins College of Engineering for Women, Pune in the year 2020 .

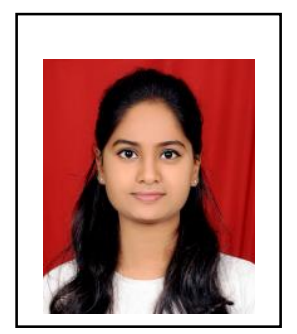

\section{Shivani Bollabattin,} Completed BTech from MKSSS's Cummins College of Engineering for Women, Pune in the year 2020 .

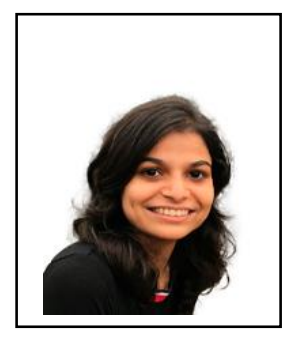

\section{Rucha Patel,} Completed BTech from MKSSS's Cummins College of Engineering for Women, Pune in the year 2020 .

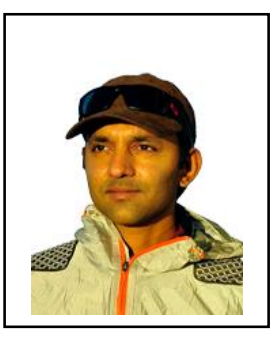

\section{Asim}

Bhalerao,

Founder \& CEO - Fluid Robotics.

Intuitive Surgical

Robotics (USA)

MSc, Mechanical

Design(USA)

MSc, Computer

Science Robotics (USA)

BE, Electronics

Engineering (India)

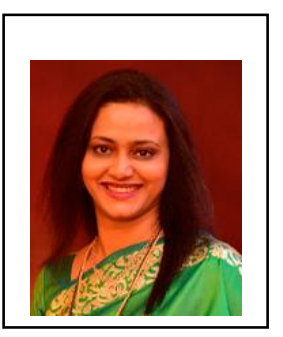

Dr. Dipti D. Patil, Associate Professor in Information

Technology

Department

of

MKSSS's Cummins College of Engineering for Women, Pune. She earned her doctorate in the year 2014 from Sant Gadge baba Amravati University in Computer Science and Engineering. She pursued her UG and 


PG Computer
Engineering from
Thadomal Shahani
Engineering College,
Bandra of Mumbai
University in 2002 and
2008 respectively. She
has authored books in
areas of Data Structures
and Mobile healthcare.
Dr. Dipti has published
many research articles
in the area of Smart
Health and Artificial
Intelligence. She is
involved in developing
healthcare system
prototypes and for
which she has filed
various national and
international patents.
Her areas of special
interest include mobile
healthcare, analytics,
data science, artificial
intelligence and
Internet of Things. She
is life member of
professional bodies like
CSI and ISTE.

